\title{
1 RNA-Seq is not required to determine stable reference 2 genes for qPCR normalization
}

3

4 Nirmal Kumar Sampathkumar*, \$, a, b, f, Venkat Krishnan Sundaram*, ${ }^{*}$, c , Prakroothi S Danthi

5 e, Rasha Barakat ${ }^{\mathrm{d}}$, Shiden Solomon ${ }^{\mathrm{a}, \mathrm{b}}$, Mrityunjoy Mondal ${ }^{\mathrm{a}, \mathrm{b}}$, Ivo Carre ${ }^{\mathrm{a}, \mathrm{b}}$, Tatiana El

6 Jalkh $^{\mathrm{c}}$, Aïda Padilla-Ferrer ${ }^{\mathrm{c}}$, Julien Grenier ${ }^{\mathrm{c}}$, Charbel Massaad ${ }^{\mathrm{c}}$, Jacqueline C Mitchell ${ }^{\mathrm{b}}$.

7

8

9 Affiliations

10 a UK-Dementia Research Institute, King's College London, London, UK.

$11 \mathrm{~b}$ Department of Basic and Clinical Neuroscience, Institute of Psychiatry, Psychology and

12 Neuroscience, Basic and Clinical Neuroscience Institute, King's College London, London 13 UK.

$14{ }^{\mathrm{c}}$ INSERM UMRS 1124, Université de Paris, Faculty of Basic and Biomedical Sciences, 15 Paris, France.

$16 \mathrm{~d}$ INSERM U1016, Institut Cochin, Paris, France.

17 e McGill Group for Suicide Studies, Douglas Mental Health University Institute, McGill 18 University, Montréal, Canada.

19 f Present address: Alzheimer's Research UK Oxford Drug Discovery Institute, Centre for 20 Medicines Discovery, University of Oxford, Oxford, UK

23 ¿Correspondence to nirmal.sampathkumar@cmd.ox.ac.uk and venkatkrishnan.sundaram@u24 paris.fr

$25 *$ These authors contributed equally. 


\section{Author contributions:}

Conceptualization: NKS, VKS.

30 Methodology: NKS, VKS

31 Experimentation: VKS, VKS, RB, SMS, IC, MM, TEJ, APF.

32 Data analysis: VKS, NKS, PSD.

33 Validation: JM, CM.

34 Visualization: JG, MM.

35 Writing - original draft: NKS, VKS.

36 Writing - review \& editing: All Authors.

\section{Acknowledgements:}

The authors thank the Animal House Core Facility and the Cyto2BM Molecular Biology Platform of BioMedTech Facilities (INSERM US36/CNRS UMS2009) for the animals and research services pertaining to the generation of qPCR data. We also thank Benjamin SAINTPIERRE and Dr. Franck LETOURNEUR from the Genomic Platform at Institut Cochin, Paris for the quality control of RNA samples and for their assistance in the analysis of RNAseq data. NKS would like to thank Prof. Christopher E SHAW and Tanisha LEWIS from the UK Dementia Research Institute, King's College London, for their support towards this work.

\section{Data availability:}

The original contributions presented in the study are included in the article \& Supplementary Data. The metadata for the RNA-Seq and qPCR experiments can be accessed at https://doi.org/10.6084/m9.figshare.15169104.v1. The datasets available include the normalized count matrices for the RNA-Seq data, qPCR Cq values and differential expression analysis, primer sequences, Coefficient of Variation and NormFinder analysis. Further inquiries can be directly addressed to the corresponding authors.

\section{Funding:}

59

60 No specific funding for the study. VKS and APF were funded by a PhD fellowship from the 61 French Ministry of Research and Innovation and Université de Paris. RB was funded by a 62 doctoral fellowship from INSERM U1016, Institut Cochin, Paris. TEJ was funded by a doctoral 63 fellowship from Université de Paris. SS and IC were funded by a PhD fellowship from the UK 64 Dementia Research Institute. MM and NKS were funded by a postdoctoral fellowship from the 65 UK Dementia Research Institute. 


\section{Abstract}

67

68 Assessment of differential gene expression by qPCR is heavily influenced by the choice of 69 reference genes. Although numerous statistical approaches have been proposed to determine 70 the best reference genes, they can give rise to conflicting results depending on experimental 71 conditions. Hence, recent studies propose the use of RNA-Seq to identify stable genes followed by the application of different statistical approaches to determine the best set of reference genes 73 for qPCR data normalization. In this study, we demonstrate that the statistical approach to determine the best reference genes from randomly selected candidates is more important than the preselection of 'stable' candidates from RNA-Seq data. Using a qPCR data normalization workflow that we have previously established; we show that qPCR data normalization using randomly chosen conventional reference genes renders the same results as stable reference genes selected from RNA-Seq data. We validated these observations in two distinct crosssectional experimental conditions involving human iPSC derived microglial cells and mouse sciatic nerves. These results taken together show that given a robust statistical approach for 81 reference gene selection, stable genes selected from RNA-Seq data do not offer any significant advantage over commonly used reference genes for normalizing qPCR assays.

\section{Keywords: qPCR, Reference Genes, RNA-Seq, Normalization Factor, Statistical} Methods 


\section{Introduction}

Normalization of relative gene expression using qPCR assays relies crucially on the use of reference genes that exhibit minimal variation across experimental conditions (Bustin et al, 2009; Thellin et al, 1999; Eisenberg \& Levanon, 2013; Jacob et al, 2013; Chapman \& Waldenström, 2015). Thus, the choice of reference genes has a significant bearing on the normalized profiles of target genes. In the last two decades, many statistical approaches have been proposed to help researchers identify stable reference genes from a given set of candidates (Vandesompele et al, 2002; Pfaffl et al, 2004; Andersen et al, 2004; De Spiegelaere et al, 2015; Silver et al, 2006; Boda et al, 2009). However, the calculations and assumptions employed in these approaches fundamentally differ and could give rise to conflicting results (Sundaram et $a l, 2019)$. To address these concerns, we recently reviewed these approaches and following a comparison of existing methods including Coefficient of Variation (CV) analysis, GeNorm, NormFinder, pairwise $\Delta C T$ method and Best Keeper, we proposed an effective qPCR analysis workflow (Sundaram et al, 2019). Our approach combining visual representation and statistical testing of intrinsic variation, $\mathrm{CV}$ analysis for identifying overall reference gene variation and the NormFinder algorithm, proved to be effective in determining stable reference genes from a given set of candidates.

However, more recently, a growing number of studies have proposed the use of bulk RNA-Seq to screen for stable reference genes followed by the application of existing statistical methods to obtain the "best" reference genes to normalize qPCR assays (Carmona et al, 2017; Zhan et al, 2014; Hoang et al, 2017; Yang et al, 2014; Chim et al, 2017; Dai et al, 2021; Pombo et al, 2017; Zhou et al; Hernandez-Segura et al, 2019). In these studies, the intrinsic validity of screening for reference genes directly from the bulk RNA-Seq data is clear, as they have used RNA-Seq to identify stable reference genes in an effort to standardize the use of certain genes for their experimental setting. Although bulk RNA-Seq data analysis pipelines quantify differential expression of target genes, performing a qPCR confirmation is often prudent as genes that exhibit shorter transcript lengths and lower expression levels exhibit discordant results between RNA-Seq and qPCR (Coenye, 2021; Everaert et al, 2017). This is largely because frequently used RNA-Seq normalization strategies are prone to overall and sample specific transcript-length bias wherein longer transcripts are attributed with more counts regardless of expression levels (Oshlack \& Wakefield, 2009; Mandelboum et al, 2019). Moreover, in a standard RNA-Seq experiment employing 3-4 biological replicates per condition, a vast majority of the reads arise from a small set of highly expressed genes and thus there is an inherent discrimination towards genes that are less expressed in the system (Berge et al, 2019; Liu et al, 2014). These factors are mostly likely at play when discordant differential expression results are obtained between qPCR and RNA-Seq (Everaert et al, 2017). Thus, the selection of qPCR reference genes from RNA-Seq data is not the best strategy as it can potentially lead to sub-optimal candidates. Moreover, the cost-benefit trade off in performing RNA-Seq to identify stable reference genes for qPCR assays is negligible. This approach is also not always feasible, especially in cases of sample scarcity or when dealing with poor yields of RNA. It is particularly relevant when we are interested in only a small number of target 
129 genes whose expression can be assessed solely by qPCR. However, although qPCR is not prone

130 to the same biases as RNA-Seq, the strongest impediment to performing reliable qPCR data 131 analysis is poor reference gene selection for a given experimental setting. Thus, the approach 132 used to validate reference genes is of utmost importance and it should ideally not be dependent 133 on a specific source of "stable" candidates. It is also advantageous if suitable reference genes 134 can be identified by the same technology rather than RNA-Seq.

In this study, we address these concerns by demonstrating that the statistical approach employed to validate reference genes is more important than the preselection of stable genes

138 from bulk RNA-Seq data. Specifically, with the right statistical approach, reference genes 139 filtered from any random set of candidates generate the same differential expression results 140 when compared to stable genes preselected from bulk RNA-Seq data. Furthermore, the results 141 obtained are also comparable with the fold changes observed in RNA-Seq for the genes that 142 we tested in qPCR. These results taken together demonstrate that the preselection of candidate 143 reference genes is not of any significance for data normalization and hence, RNA-Seq data is 144 not an essential requisite to obtain robust reference genes for qPCR data normalization. 


\section{Materials and Methods:}

\section{Sample Procurement:}

iPSC Microglia:

150 The CRISPR/Cas gene edited TREM2 knock-out iPSC line BIONi010-C-17 (TREM2 KO) and its isogenic control BIONi010-C (TREM2 WT) were purchased from EBISC and maintained at $37^{\circ} \mathrm{C} 5 \% \mathrm{CO} 2$ with E8-Flex medium. Microglia were differentiated from these lines as previously described (Haenseler et al, 2017). In short, using AggreWell 800 (34850, StemCell Technologies), embryonic bodies (EBs) were prepared from iPSCs in E8-Flex medium supplemented with $50 \mathrm{ng} / \mathrm{ml}$ VEGF (PHC9394, ThermoFisher), $50 \mathrm{ng} / \mathrm{ml}$ BMP4 (120-05ET, Peprotech), and $20 \mathrm{ng} / \mathrm{ml} \mathrm{SCF}$ (300-07, PeproTech) for 3 days with $75 \%$ medium change each day. EBs were transferred to a T75 flask in X-VIVO15 (BE02-060F, Lonza) medium supplemented with $25 \mathrm{ng} / \mathrm{ml}$ IL-3 (PHC0031, ThermoFisher), $2 \mathrm{mM}$ Glutamax (35050061, ThermoFisher), $100 \mathrm{ng} / \mathrm{ml} \mathrm{M-CSF}$ (300-25, PeproTech), and $0.055 \mathrm{mM} \beta$-mercaptoethanol (31350-010, ThermoFisher). Precursor cells started to emerge from the EBs approximately 4 weeks later, and were collected by gently tapping the flask. These precursor cells are differentiated to microglia over 7-days in DMEM F:12, Neuronal basal medium plus $(1: 1)$ supplemented with $100 \mathrm{ng} / \mathrm{ml} \mathrm{IL-34,} 10 \mathrm{ng} / \mathrm{ml} \mathrm{GM-CSF}$, and $100 \mathrm{ng} / \mathrm{ml} \mathrm{M-CSF}$.

\section{Sciatic Nerves:}

To assess the stability of commonly used reference genes during myelination of the sciatic nerves, P3 and P21 C57BL6/J mice (3 Males and 4 females) were dissected and the sciatic nerves were harvested for RNA extraction. The RNA-seq data for these time points was mined from a publicly available dataset as explained in the "Bulk RNA-seq and data analysis" section below. All aspects of animal care and animal experimentation were performed in accordance with the relevant guidelines and regulations of INSERM, Université de Paris, and approved by the French National Committee of Animal experimentation and ethics.

\section{Total RNA extraction :}

177 Approximately $2 \times 10^{5}$ iPSC derived microglial cells were harvested using TRIzol reagent 178 (Ambion Life Technologies 15596018) for total RNA isolation. Total RNA was isolated using 179 Direct-Zol ${ }^{\mathrm{TM}}$ RNA microprep columns (Zymo research R2062) according to the manufacturer's protocol.

\section{Sciatic Nerves:}

183 Total RNA was extracted from sciatic nerves using $1 \mathrm{~mL}$ of TRIzol reagent (Ambion Life 184 Technologies 15596018) on ice using the manufacturer's instructions with slight modifications. Briefly, $100 \%$ ethanol was substituted for isopropanol to reduce the precipitation of salts. In addition, RNA precipitation was carried out overnight at $-20^{\circ} \mathrm{C}$ in the presence of glycogen $(0.02 \mathrm{mg} / \mathrm{mL}$ final concentration). The following day, precipitated RNA 
was pelleted by centrifugation and washed at least 3 times with 70\% Ethanol to eliminate any residual contamination. Tubes were then spin-dried in a vacuum centrifuge for 5 minutes and RNA was resuspended in $20 \mu \mathrm{L}$ of RNase resuspension buffer containing $0.1 \mathrm{mM}$ EDTA, pH 8. RNA was then stored at $-80^{\circ} \mathrm{C}$ till RTqPCR.

\section{RNA Quality, Integrity, and Assay:}

\section{iPSC Microglia:}

RNA quantity was assayed using UV spectrophotometry on Nanodrop One (Thermo Scientific). Optical density absorption ratios A260/A280 \& A260/A230 of the iPSC microglia samples were $2.0( \pm 0.1 \mathrm{SD})$ and $2.1( \pm 0.1 \mathrm{SD})$ respectively. RNA integrity was verified using Agilent bioanalyzer. All the samples exhibited a RIN score $\geq 9$ and were subsequently used for downstream analysis including paired-end bulk RNA-seq and qPCR.

\section{Sciatic Nerves:}

RNA quantity was assayed using UV spectrophotometry on Nanodrop One (Thermo Scientific). Optical density absorption ratios A260/A280 \& A260/A230 of sciatic nerve samples were $1.84( \pm 0.04 \mathrm{SD})$ and $1.54( \pm 0.53 \mathrm{SD})$ respectively. RNA integrity was verified using Agilent bioanalyzer at the Genomics Platform at Institut Cochin, Paris. All samples exhibited a RIN score $\geq 8.8$ and were used for qPCR analysis.

\section{Bulk RNA-seq and data analysis:}

For the iPSC samples, total RNA was extracted and sent to Genewiz, Germany for paired-end bulk RNA-seq. The raw data, final processed data and the metadata are available in the GEO database (GSE178924). For the sciatic nerves, bulk RNA-Seq data was obtained from a previously published study (Ackerman $e t a l, 2018$ ). The raw data can be accessed at the Zenodo repository (https:// zenodo.org/record/1154250). Only the WT datasets at P3 and P21 were mined and re-analyzed. Fastq files were aligned using the STAR algorithm (version 2.7.6a). Reads were then counted using RSEM (v1.3.1) and the statistical analyses on the read counts were performed with the DESeq2 package (DESeq2_1.28.1) to determine the proportion of differentially expressed genes between the two experimental conditions (Love et al, 2014). The standard DESeq2 normalization method (DESeq2's median of ratios with the DESeq function) was used, with a pre-filter of reads and genes (reads uniquely mapped on the genome, or up to 10 different loci with a count adjustment, and genes with at least 10 reads in at least 3 different samples). The biomaRt package (v2.44.4) was used to substitute gene names for the Ensembl IDs in the count matrix (Durinck et al, 2009). The DESeq2 pipeline was used to fit a generalized linear model (GLM) for the expression of each gene relative to the experimental groups. To determine the variation in gene expression, the dispersion value computed by the GLM was tabulated into the results data frame. Square root of dispersion was calculated using sqrt function from base functions in R (v4.0.2). The coefficient of dispersion (CVfromDisp) was calculated by multiplying square root of dispersion by 100 . 


\section{Reference gene selection from RNA-Seq:}

The data frame was subjected to 4 filtration methods to select stable genes in both the iPSC and Sciatic nerve datasets. First filtration was done by retaining all the genes with padj value above 0.05 . The resulting data frame was ordered based on the $\log 2$ FoldChange $(\log 2 \mathrm{FC})$ column and the second filtration was performed by retaining all the genes with $\log 2 \mathrm{FC}$ between -0.1 to 0.1 implying negligible intergroup variation. Third filtration was done by retaining all the genes that lie in the CVfromDisp range between $10 \& 20$. The fourth and last filtration was performed by retaining all the genes that exhibited a Basemean value greater than or equal to 500 implying sufficient expression for qPCR detection.

Once these criteria were applied, 683 genes were obtained (basemean values ranging from 500 to 123000 ) in the iPSC dataset and 42 genes were obtained (basemean values ranging from 500 to 48000) in the sciatic nerve dataset. To obtain stable reference genes with both low and high expression, the final list of candidates was partitioned into quartiles based on basemean expression. 3 reference genes from each of the first three quartiles and 1 reference gene from the last quartile were chosen; thereby generating 10 candidates. This selection strategy resulted in candidate reference genes that exhibit $\mathrm{Cq}$ values between 18 cycles (high expression) and 26 cycles (relatively lower expression) in qPCR.

\section{Conventional reference gene selection:}

All conventional reference genes were randomly chosen. They do not have any proven stability for the experimental setting in question. For the iPSC dataset, the conventional human reference genes chosen were ACTB, GAPDH, GUSB, HPRT, PGK1, PPIA, RPL13A, TBP, and $U B C$. For the sciatic nerve dataset, the conventional mouse reference genes chosen were $A c t b$, Gapdh, Tbp, Sdha, Pgk1, Ppia, Rpl13a, Hsp60, Mrpl10, Rps26. These mouse reference genes have been previously used to establish the qPCR data analysis workflow (Sundaram et al, 2019)

\section{Target gene selection:}

Differentially expressed genes that exhibited Padj $<0.05$ were first retained from the results dataframe. Next, genes that exhibited $\log 2 \mathrm{FC}$ values above +0.6 and below -0.6 were retained and partitioned into 2 separate lists. 3 target genes were randomly chosen from each of these lists for differential expression analysis in the iPSC dataset. In the Sciatic nerve dataset, 3 upregulated genes and 3 downregulated genes were chosen based on the recently published Sciatic Nerve Atlas https://www.snat.ethz.ch (Gerber et al, 2021).

\section{Primer Design:}

All primers used in the study were designed using the Primer 3 plus software (https://primer3plus.com/cgi-bin/dev/primer3plus.cgi). Splice variants and the protein-coding sequence of the genes were identified using the Ensembl database (www.ensembl.org). 
Constitutively expressed exons among all splice variants were then identified using the ExonMine database (Mollet et al, 2010). Primers that spanned two subsequent constitutively expressed exons were then designed using the Primer 3 plus software. The amplicon size of all primers was between $88 \mathrm{bp}-200 \mathrm{bp}$. For detailed information on Primer sequences refer to Supplementary data.

\section{Amplification Efficiencies:}

283

The amplification efficiencies of primers were calculated using serial dilution of cDNA molecules. Briefly, cDNA from both the experimental groups of the sciatic nerve (for mouse primers) and iPSC (for human primers) were serially diluted four times by a factor of 10 (1, $1: 10,1: 100 \& 1: 1000)$. qPCR was then performed using these dilutions and the results were plotted as a standard curve against the respective concentration of cDNA. Amplification efficiency (E) was calculated by linear regression of standard curves using the following

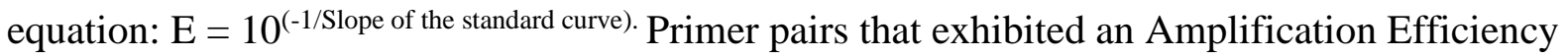
(E) of 1.9 to $2.1(95 \%-105 \%)$ and an $\mathrm{R}^{2}$ value (Determination Coefficient) of 0.99 or above were chosen for this study. The $\mathrm{Cq}$ values of all genes across different groups were well within the range of the standard dilution curve.

\section{RT-qPCR:}

\section{iPSC Microglia:}

$1000 \mathrm{ng}$ of total RNA was first subjected to DNase digestion (Thermo scientific EN0525) at $37^{\circ} \mathrm{C}$ for 30 minutes to eliminate contaminating genomic DNA. Next, DNase activity was stopped using EDTA (Thermo scientific) and cDNA synthesis was done using iSCRIPT ${ }^{\mathrm{TM}}$ cDNA synthesis kit from BioRad (1708891) to a total volume of $20 \mu 1$. cDNA was diluted 1:10 with nuclease free water for qPCR analysis. qPCR was performed using Takyon ROX SYBR 2X MasterMix (Eurogentec UF-RSMT-B0701) as a fluorescent detection dye. All reactions were carried out in a final volume of $8 \mu \mathrm{l}$ in 384 well plates with $300 \mathrm{nM}$ gene-specific primers, around $5 \mathrm{ng}$ of cDNA (at 100\% RT efficiency) and 1X SYBR Master Mix in each well. Each reaction was performed in triplicates. All qPCR experiments were performed using ThermoFisher Scientific QuantStudio 7 with a No-Template-Control (NTC) to check for primer dimers and a No-RT-Control (NRT) to check for any genomic DNA contamination.

\section{Sciatic Nerves:}

500 ng of total RNA was reverse transcribed with Random Primers (Promega C1181) and MMLV Reverse Transcriptase (Sigma M1302) according to prescribed protocols. qPCR was performed using Absolute SYBR ROX 2X qPCR mix (Thermo AB1162B) as a fluorescent detection dye. All reactions were carried out in a final volume of $7 \mu 1$ in 384 well plates with $300 \mathrm{nM}$ gene-specific primers, around $3.5 \mathrm{ng}$ of cDNA (at 100\% RT efficiency) and 1X SYBR Master Mix in each well. Each reaction was performed in triplicates. All qPCR experiments 
319 were performed on BioRad CFX384 with a No-Template-Control (NTC) to check for primer

320 dimers and a No-RT-Control (NRT) to check for any genomic DNA contamination.

\section{qPCR statistical analysis and data visualization:}

qPCR readouts were analyzed in Precision Melt Analysis Software v1.2 (Sciatic nerve samples) and QuantStudio Real-Time PCR Software v1.7.1 (iPSC samples). The amplicons were subjected to Melt Curve analysis and were verified for a single dissociation peak at a Melting Temperature $(\mathrm{Tm})>75^{\circ} \mathrm{C}$ as expected from the primer constructs. The Cq data was exported to Microsoft Excel for further calculations. Each biological sample had three technical replicates thereby generating three individual $\mathrm{Cq}$ values. The arithmetic mean of the triplicates was taken to be the $\mathrm{Cq}$ representing the biological sample. The standard deviation (SD) of the triplicates was also calculated and samples that exhibited SD > 0.20 were considered inconsistent. In such cases, one outlier $\mathrm{Cq}$ was removed to have at least duplicate $\mathrm{Cq}$ values for each biological sample and an SD $<0.20$.

Reference gene validation was performed according to our qPCR data analysis workflow (Sundaram et al, 2019). Visual representation of potential intrinsic variation in reference genes was identified by plotting the raw expression profiles $\left(2^{-\Delta \mathrm{Cq}}\right)$ of all candidate reference genes. Due to reduced sample sizes, a non-parametric Mann Whitney U-Test was performed to assess statistically significant expression variation between experimental groups. The alpha value was set at 0.05 for statistical significance. The genes were then screened using Coefficient of variation $(\mathrm{CV})$ analysis to eliminate genes that exhibited $\mathrm{CV}>50 \%$ as they could impede the robustness of the Normfinder algorithm (Sundaram et al, 2019). Normfinder was then used to determine the best pair of reference genes (least $\mathrm{S}$ value) to compute the Normalization Factor (NF) for qPCR data normalization (Andersen et al, 2004). From the resulting pair of stable reference genes, the NF was then calculated as the arithmetic mean of the Cq values of the 2 genes for each sample. Relative expression of target genes was quantified using the $2^{-\Delta \Delta \mathrm{Ct}}$ method using the NF as the reference gene (Schmittgen \& Livak, 2008; Livak \& Schmittgen, 2001). Data was then analysed and visualised using R studio. For the fold change comparison between qPCRs and RNA-Seq, the non-parametric Kruskal-Wallis ANOVA was performed. Multiple group comparisons were performed using the non-parametric Dunn's Post Test. The alpha value was set at 0.05 for statistical significance. 


\section{Results:}

\section{Reference gene selection from RNA-Seq of microglia (WT vs TREM2KO)}

A growing body of literature promotes the use of RNA-Seq to identify stable reference genes for qPCR data normalization in different experimental conditions. However, we believe that this approach is superfluous and that stable reference genes can readily be identified by starting out with any random set of candidates and adopting the right statistical approach to determine the best reference genes for a given experimental condition. To test this hypothesis, we first performed paired-end bulk RNA-Seq on iPSC-derived WT and TREM2KO microglia, an experimental model used to study the role of microglia in neuroinflammation and neurodegeneration (McQuade et al, 2020; Andreone et al, 2020). Based on the criteria detailed in the Materials and Methods section (see Reference gene selection from RNA-Seq), we shortlisted 10 candidate reference genes (ANXA7, APIP, CCNT2, CNBP, DDX42, FOXK1, KIF13A, MRPL37, PPP1R10, and USP5) from our RNA-Seq data. The base mean values of these genes and other RNA-Seq data features are detailed in Supplementary Table 1.

\section{Reference gene validation of RNA-Seq derived reference genes (WT vs TREM2KO)}

The reference genes selected from RNA-Seq were then subjected to validation using the qPCR workflow that we developed recently (Sundaram et al, 2019). We first computed the intrinsic variation of reference genes by linearizing the $\mathrm{Cq}$ values $\left(2^{-\mathrm{Cq}}\right)$ followed by visually representing the non-normalized expression levels $\left(2^{-\Delta \mathrm{Cq}}\right)$ of the genes in both experimental groups (Figure 1). The WT group was used as the experimental calibrator. Subsequently, statistical testing was performed using a non-parametric Mann Whitney Test to determine significant differences between the groups. Among the 10 genes tested, only 2 genes (MRPL37 WT and TREM2KO groups (Figure 1). 

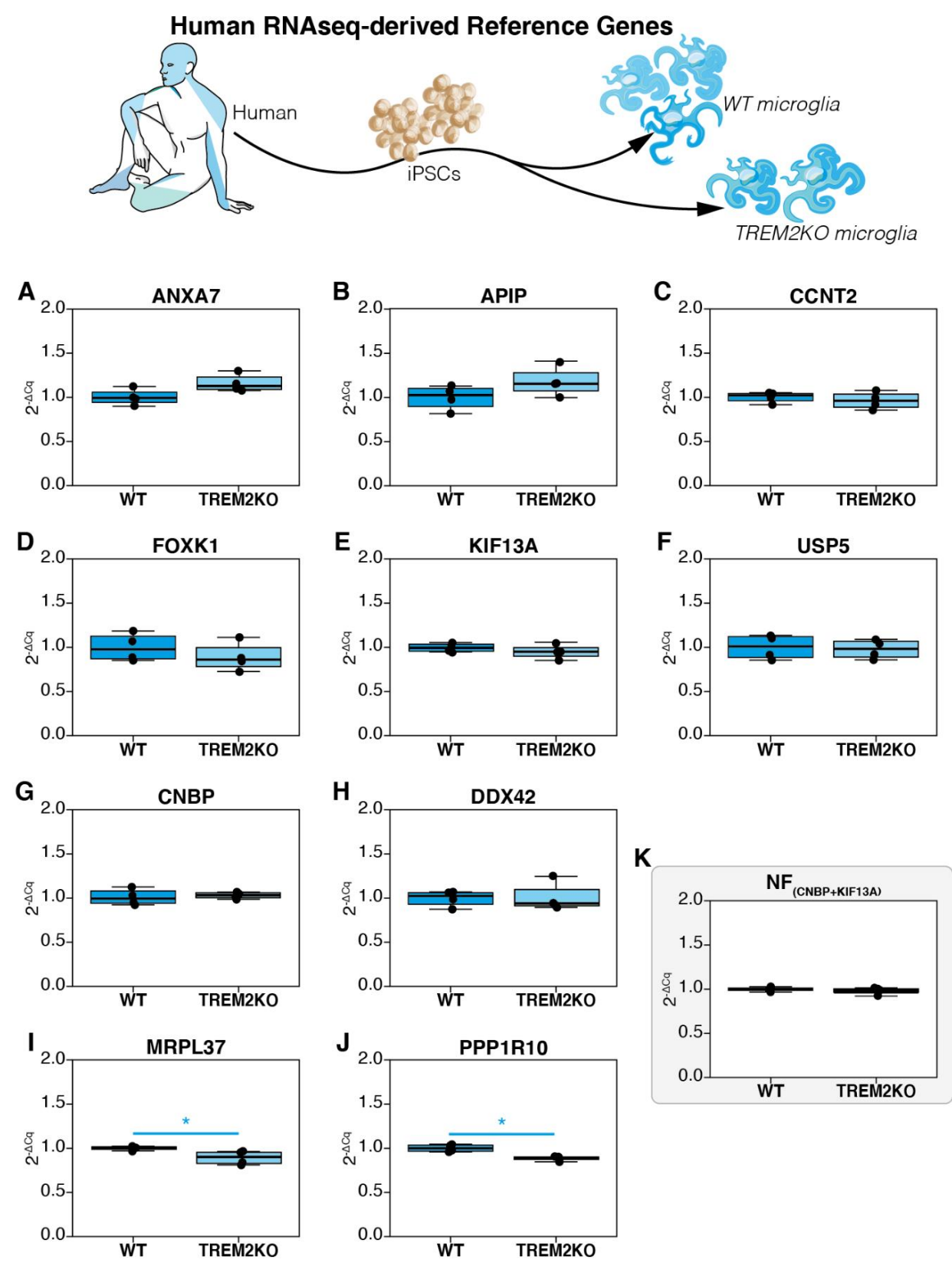

Figure 1. Non-normalized expression profiles $\left(2^{-4 C q}\right)$ of reference genes derived from RNA-

381 Seq data of iPSC derived microglia. WT group was used as the experimental calibrator. (a) ANXA7, (b) APIP, (c) CCNT2, (d) FOXK1, (e) KIF13A, (f) USP5, (g) CNBP, (h) DDX42, (i) MRPL37, (j) PPIRIO and ( $k$ ) Normalization Factor (NF) assessed by NormFinder that combines CNBP \& KIF13A. Non-parametric Mann Whitney $U$ test was used to assess differences between the groups. The alpha value was set at 0.05 and $P$ values are annotated as follows: $* P<0.05$.

In accordance with our workflow, we next performed Coefficient of Variation analysis on linearized $\mathrm{Cq}$ values $\left(2^{-\mathrm{Cq}}\right)$ to assess the overall variation (both groups included) of the reference genes (Table 1). The genes tested exhibited CV values between 5.99\% and $15.75 \%$ and they were ranked as shown in Table 1. As all genes exhibited $\mathrm{CV}<50 \%$, they could therefore be screened using the NormFinder algorithm to determine the best combination of 
392 reference genes to be used for data normalization. This is a crucial step of reference gene

393 validation as genes that exhibit $\mathrm{CV}>50 \%$ can compromise the robustness of the algorithm

394 (Sundaram et al, 2019). NormFinder determines the stability of a reference gene by factoring

395 the intergroup as well as the intragroup variation and computes a Stability S Score (Andersen et al, 2004). The lower the $S$ score, the higher the stability of the gene across all experimental groups. The algorithm also suggests the best pair of reference genes that can be used for robust data normalization. NormFinder results of the selected reference genes from RNA-seq are represented in Table 1 along with their respective $S$ Scores and ranks. The algorithm further revealed CNBP and KIF13A to be the best combination of genes with a grouped Stability S score of 0.05 (Table 1). The $\mathrm{Cq}$ values of these two genes were then combined to compute the Normalization Factor (NF). Evidently and as expected, the non-normalized expression level of the NF does not vary significantly between the two groups (Figure 1K). It is to be noted that the combination of the two best reference genes exhibits lesser intergroup and intragroup variation (Figure 1K versus the rest) including the top ranked CNBP (Figure 1G). This is an inherent feature of the algorithm.

407 Table 1. CV and NormFinder analysis

408 Expression stability of candidate reference genes derived from bulk RNA-Seq data in 409 iPSC-derived microglia were evaluated using Coefficient of Variation (CV) Analysis \& 410 NormFinder.

\begin{tabular}{|l|c|c|l|c|c|}
\hline \multicolumn{1}{|c|}{ CV Analysis } & \multicolumn{3}{c|}{ NormFinder } \\
\hline Gene & $\%$ CV & Rank & Gene & Stability S & Rank \\
\hline CNBP & 5.99 & 1 & CNBP & 0.07 & 1 \\
\hline KIF13A & 6.36 & 2 & CCNT2 & 0.08 & 2 \\
\hline PPP1R10 & 6.87 & 3 & KIF13A & 0.1 & 3 \\
\hline CCNT2 & 7.46 & 4 & PPP1R10 & 0.1 & 4 \\
\hline MRPL37 & 7.74 & 5 & USP5 & 0.1 & 5 \\
\hline ANXA7 & 10.58 & 6 & MRPL37 & 0.11 & 6 \\
\hline USP5 & 10.81 & 7 & DDX42 & 0.12 & 7 \\
\hline DDX42 & 11.41 & 8 & ANXA7 & 0.15 & 8 \\
\hline
\end{tabular}




\begin{tabular}{|l|c|c|l|c|c|}
\hline APIP & 14.61 & 9 & FOXK1 & 0.15 & 9 \\
\hline FOXK1 & 15.75 & 10 & APIP & 0.16 & 10 \\
\hline
\end{tabular}

413 Normfinder Best Pair: CNBP/KIF13A

414 Grouped Stability: 0.05

415

416 Reference gene validation of conventional human reference genes (WT vs 417 TREM2KO)

418 In addition to the reference genes derived from the RNA-Seq data, we next sought to validate

419 commonly used human reference genes chosen arbitrarily. We selected 10 reference genes 420 (ACTB, GAPDH, GUSB, PPIA, RPLPO, TBP, HPRT, PGK1, UBC, and RPL13A) and assessed 421 their intergroup variation by plotting their non-normalized profiles (Figure 2). This was 422 followed by statistical testing for significant differences between the two experimental groups. 423 Among the 10 genes tested, none of the genes exhibited significant variation in their non424 normalized expression levels (Figure 2). 

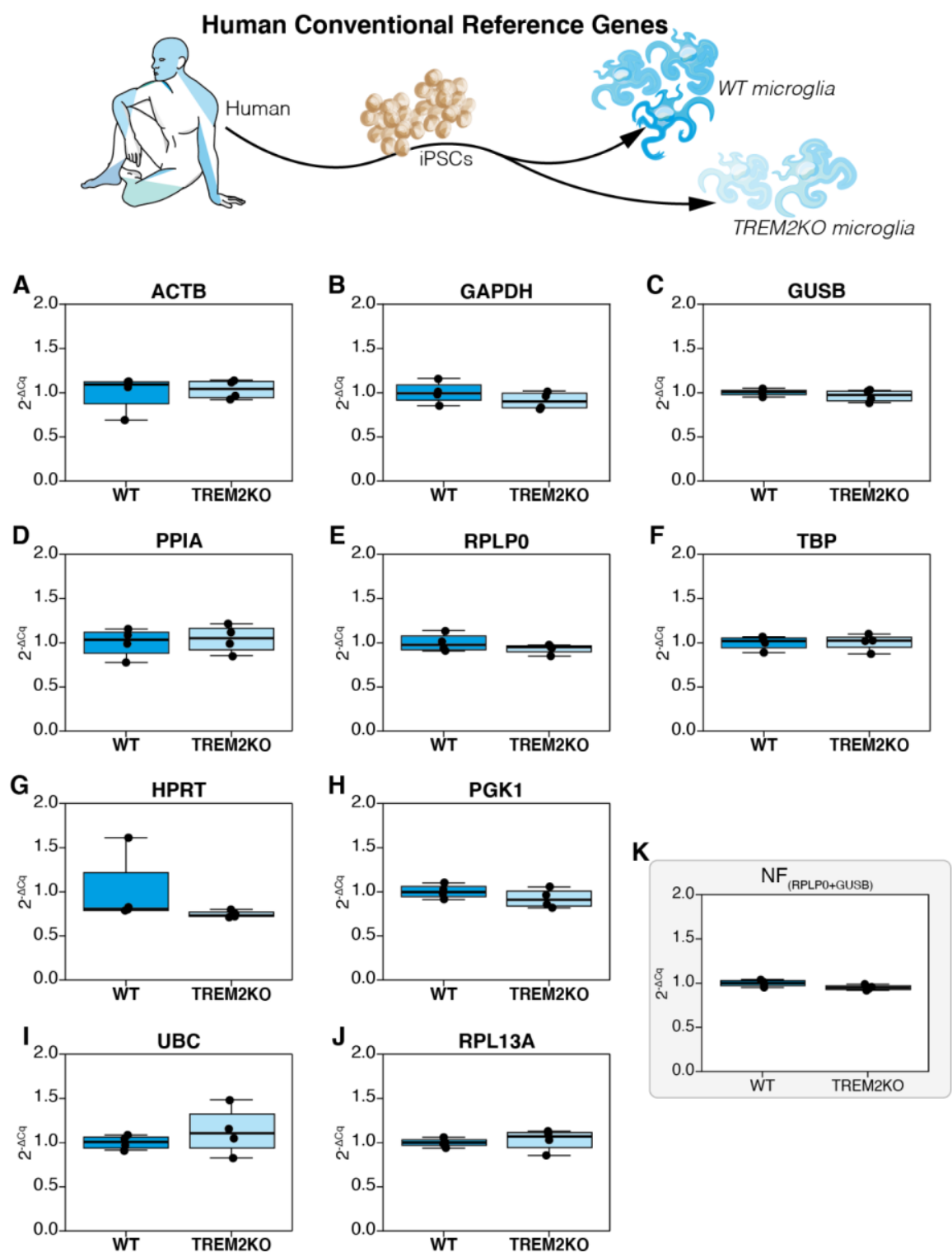

426 Figure 2. Non-normalized expression profiles $\left(2^{-\Delta C q}\right)$ of conventional human reference genes in iPSC derived microglia. WT group is used as the experimental calibrator. (a) $A C T B$, (b) GAPDH, (c) GUSB, (d) PPIA, (e) RPLPO, (f) TBP, (g) HPRT, (h) PGK1, (i) UBC, (j) RPL13A, and (k) Normalization Factor (NF) assessed by NormFinder that combines RPLPO \& GUSB. Non-parametric Mann Whitney $U$ test was used to assess differences between the groups. The alpha value was set at 0.05 and $P$ values are annotated as follows: $* P<0.05$, ** $P<0.01$, *** $P<0.001$.

433 These genes were then subjected to CV analysis to assess the overall variation (Table 2). They exhibited CV values ranging from $5.29 \%$ to $32.37 \%$. However, all genes satisfied the CV $<50 \%$ criterion for NormFinder screening. NormFinder results for these genes are also shown in Table 2. Interestingly and in contrast to the reference genes derived from the RNA-Seq data

437 (Table 1), the Stability S score of the conventional genes are largely lower than the genes derived from RNA-Seq, thus indicating better stability. The first 6 ranks from RPL13A to 
439 GAPDH have S scores less than 0.1 whereas in the previous dataset (Table 1), only the first 2 440 ranks (CNBP \& CCNT2) exhibit scores less than 0.1. Finally, among all the conventional reference genes screened, NormFinder suggested the use of RPLP0 and GUSB as the best pair 442 for data normalization with a grouped stability of 0.03 (Table 2); which in comparison, is lesser than the Stability Score of 0.05 from CNBP and KIF13A (Table 1). In principle, these results show that randomly selected reference genes could potentially exhibit better stability than stable reference genes filtered from RNA-Seq data.

Table 2: CV and NormFinder analysis of conventional reference genes. Expression stability of randomly chosen candidate reference genes were evaluated using Coefficient of Variation (CV) Analysis, NormFinder. Best pair chosen is RPLPO and GUSB with a Stability S score of 0.03 .

\begin{tabular}{|c|c|c|c|c|c|}
\hline \multicolumn{3}{|c|}{ CV Analysis } & \multicolumn{3}{|c|}{ NormFinder } \\
\hline Gene & $\% \mathrm{CV}$ & Rank & Gene & Stability S & Rank \\
\hline$G U S B$ & 5.29 & 1 & $R P L 13 A$ & 0.03 & 1 \\
\hline$T B P$ & 7.56 & 2 & $G U S B$ & 0.04 & 2 \\
\hline RPL13A & 8.16 & 3 & $R P L P O$ & 0.05 & 3 \\
\hline$R P L P O$ & 8.18 & 4 & $T B P$ & 0.05 & 4 \\
\hline$P G K 1$ & 9.56 & 5 & $P G K 1$ & 0.06 & 5 \\
\hline$G A P D H$ & 11.35 & 6 & GAPDH & 0.07 & 6 \\
\hline PPIA & 13.75 & 7 & PPIA & 0.10 & 7 \\
\hline$A C T B$ & 14.31 & 8 & $U B C$ & 0.11 & 8 \\
\hline$U B C$ & 17.47 & 9 & $A C T B$ & 0.14 & 9 \\
\hline$H P R T$ & 32.27 & 10 & $H P R T$ & 0.18 & 10 \\
\hline
\end{tabular}




\section{Differential expression of target genes by qPCR and RNA-Seq (WT vs 455 TREM2KO)}

456 Following reference gene validation of conventional and RNA-Seq-derived candidates, we 457 picked 6 target genes that were differentially expressed based on the RNA-Seq results (see 458 Target gene selection from Materials \& Methods). We included 3 target genes (TREM2, $459 P 2 R Y 12$ and CD52) that were significantly downregulated and 3 others (ACSS3, SYDE1 and $460 T B X 10$ ) that were significantly upregulated. The relative expression of these genes was then 461 computed by qPCR using the Normalization Factor (NF) from conventional candidate 462 reference genes (RPLPO \& GUSB or Conventional NF) or from RNA-Seq-derived candidate 463 reference genes (CNBP \& KIF 13A or RNA-Seq derived NF) (Figure 3). To compare our qPCR 464 results, we also computed the fold change assessed by RNA-Seq using the Normalized counts 465 of these genes.

466 Regarding the downregulated genes, TREM2 expression in the TREM2KO group, when assessed through qPCRs, exhibited expression fold change values of $0.31 \pm 0.05$ (mean FC \pm $\mathrm{SD}$, conventional NF, Figure 3A) and $0.35 \pm 0.04$ (RNA-Seq derived NF, Figure 3B). In RNASeq, the fold change values were $0.23 \pm 0.03$ (Figure 3C). $P 2 R Y 12$ fold change values in the TREM2KO group were $0.04 \pm 0.02$ when assessed using qPCRs (both conventional NF and RNA-Seq derived NF, Figure 3D and 3E). However, RNA-Seq fold change values of $P 2 R Y 12$ were comparatively higher at $0.62 \pm 0.19$ (Figure 3F). CD52 fold change in the TREM2KO group when assessed by qPCR were $0.38 \pm 0.06$ (Conventional NF, Figure 3G) and $0.40 \pm$ 0.05 (RNA-Seq derived NF, Figure 3H). RNA-Seq fold changes were comparatively lower at $0.16 \pm 0.01$ (Figure 3I).

For upregulated genes, ACSS3 in the TREM2KO group exhibited fold change values of $3.72 \pm$ 0.79 (Conventional NF, Figure 3J) and $3.40 \pm 0.88$ (RNA-Seq derived NF, Figure 3K). The fold changes in RNA-Seq were $4.54 \pm 1.25$ (Figure 3L). SYDE1 fold change values assessed by qPCR were at $3.58 \pm 1.18$ (Conventional NF, Figure 3M) and $3.29 \pm 1.28$ (RNA-Seq derived NF, Figure 3N). RNA-Seq expression levels of SYDE1 were at $3.62 \pm 0.34$ (Figure 30). Finally, TBX10 expression fold changes assessed by qPCR were at $3.06 \pm 1.51$ fold changes were at $3.24 \pm 0.61$ (Figure 3R). These results taken together with the visual representation of differential expression described in Figure 3 show that qPCR normalization using conventional NF or RNA-Seq derived NF render the same results. They do not always 

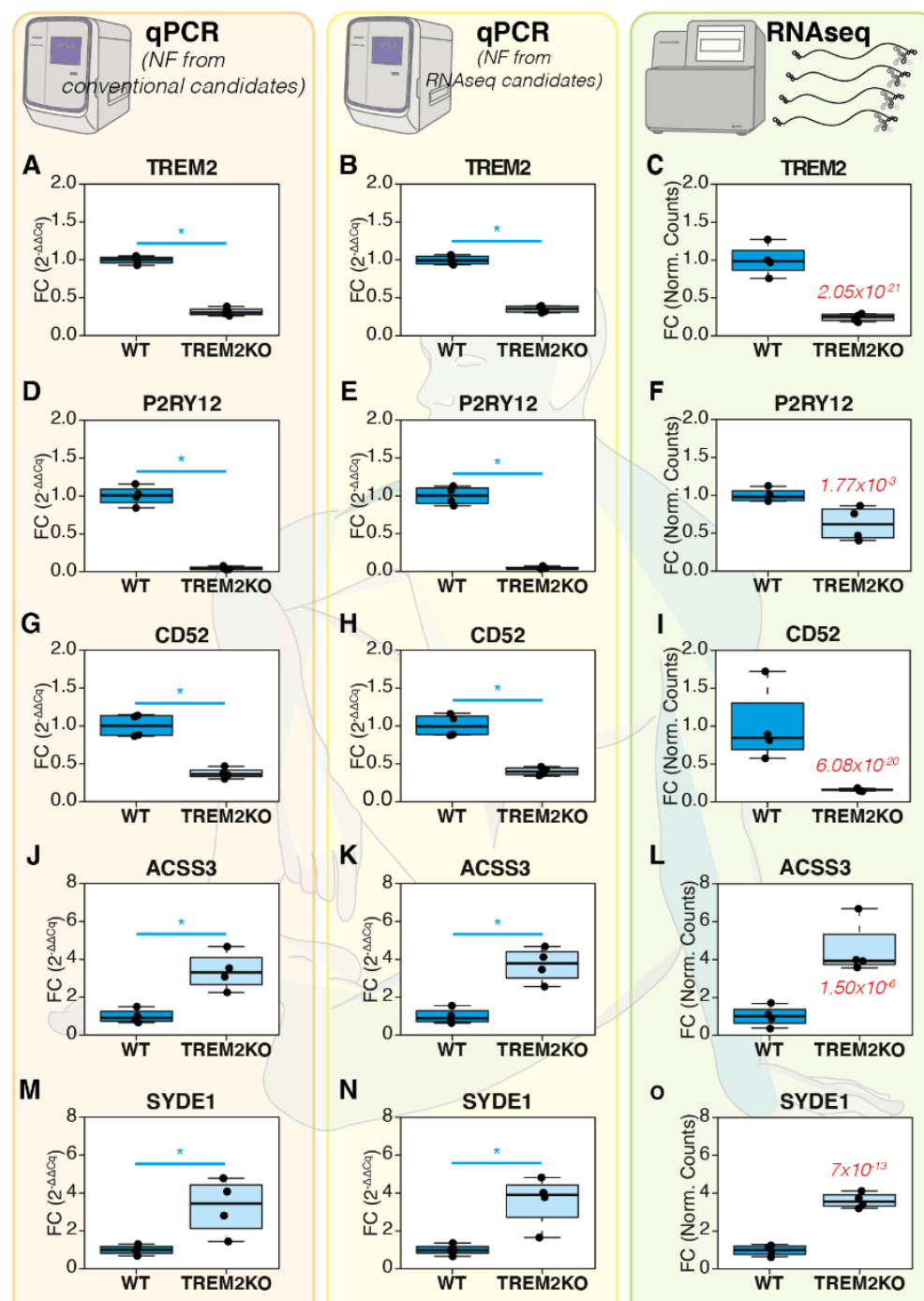

$\mathbf{N}$
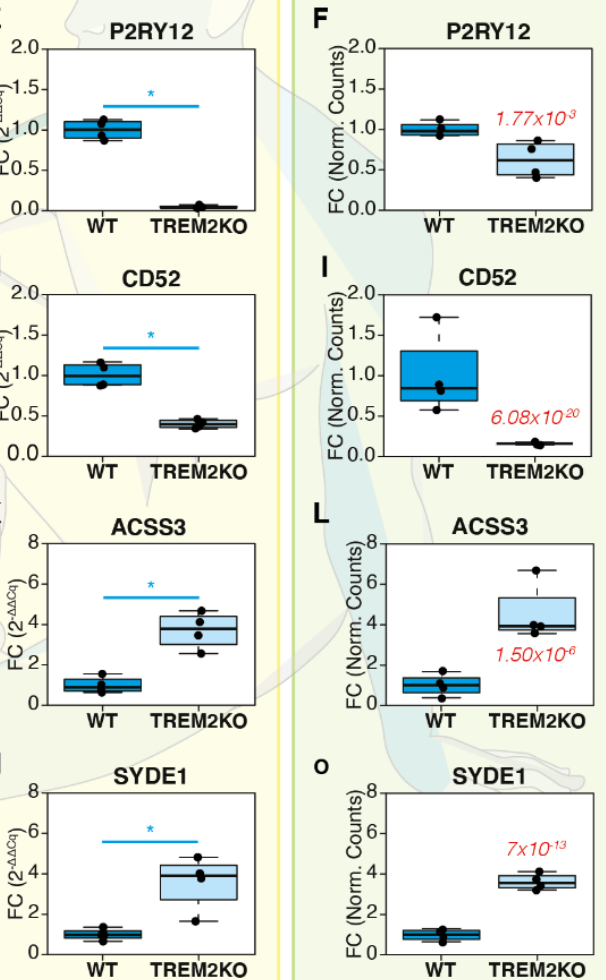

$\mathbf{L}$
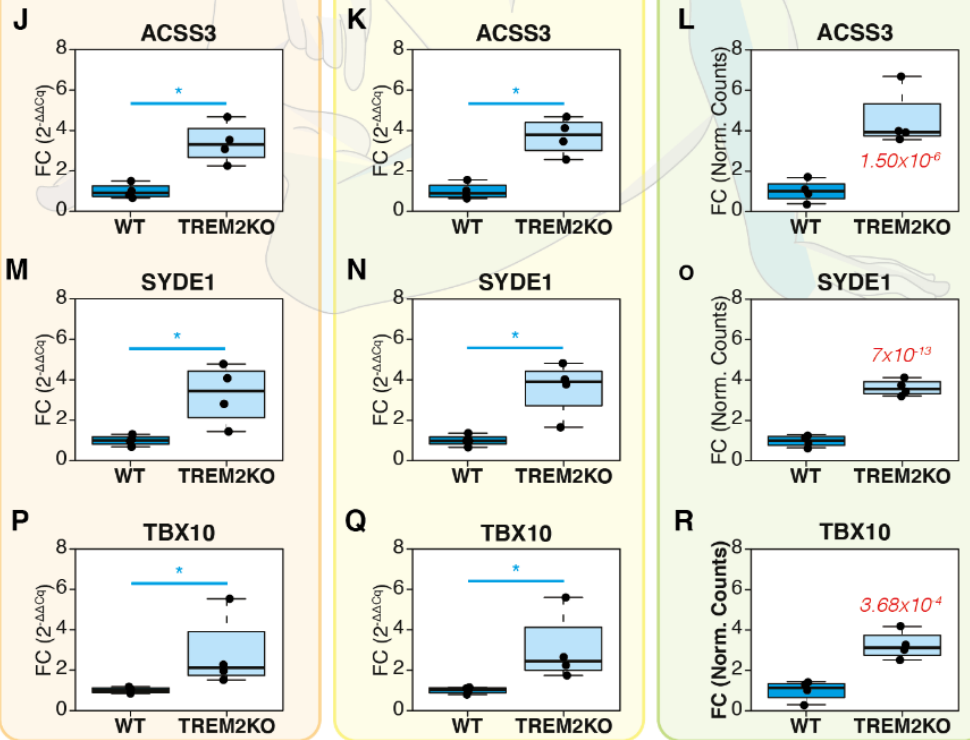

489 Figure 3: Differential expression of target genes between WT and TREM2KO iPSC cells assessed by qPCRs and RNA-Seq. The target genes (TREM2, P2RY12, CD52, ACSS3, $S Y D E 1, T B X 10)$ have been normalized with the normalization factor (NF) computed from conventional reference genes RPLPO \& GUSB in the first column $(A, D, G, J, M, P)$ or from $R N A-S e q$ derived reference genes $C N B P \& K I F 13 A$ in the second column $(B, E, H, K, N, Q)$. The third column contains the Fold Changes computed from RNA-Seq $(C, F, I, L, O, R)$. The normalized counts for each gene in each group were used to calculate the fold changes in RNASeq. The WT group was used as the experimental control. The Padj value of RNA-Seq is indicated in the TREM2KO group for each gene in the RNA-Seq column. For the qPCRs, Nonparametric Mann Whitney U test was used to assess differences between the groups. The alpha value was set at 0.05 and $P$ values are annotated as follows: $* P<0.05$. 


\section{Comparison of fold changes between qPCRs and RNA-Seq (WT vs TREM2KO):}

501 We next investigated in detail if the Fold Change distributions in the TREM2KO experimental 502 group computed across the 3 methods differed significantly from one another. To this end, we 503 compared the distributions by a non-parametric ANOVA (Kruskal Wallis Test) of ordinal 504 distributions followed by Dunn's multiple comparison post-test (Supplementary Figure 1). qPCR Fold changes computed using Conventional NF were used as the control for multiple comparisons. Therefore, the first comparison was made between the conventional and RNASeq derived NF groups (difference in qPCRs, hereafter referred to as Comparison 1) and the second comparison was made between the Conventional NF group and RNA-Seq differential expression (difference between qPCR and RNA-Seq, hereafter referred to as Comparison 2).

510 The WT experimental group was omitted from this analysis as it was used as the experimental

511 calibrator for differential expression. The mean Fold Change of the WT group is always at 1 512 regardless of the gene/method in question and therefore it is redundant to test for statistical 513 significance of the WT fold change levels across different methods for each gene.

514 Non-parametric ANOVA revealed no significant difference between the FC distributions of 515 the upregulated genes $A C S S 3$, SYDE1 and TBX1 (Supplementary Figure 1D, 1E and 1F) 516 suggesting that the qPCR fold changes concur with RNA-Seq fold changes in these genes. As 517 for the downregulated genes, TREM2 FC distributions varied significantly between the groups 518 (Kruskal Wallis P $<0.5$ ); however, Dunn's post test revealed no significant difference in 519 Comparison 1 and Comparison 2 (Supplementary Figure 1A). P2RY12 FC distributions 520 varied significantly between the groups (Kruskal Wallis $\mathrm{P}<0.01$ ). Multiple comparisons revealed no difference in Comparison 1 but a significant difference in Comparison 2 (Dunn's Post Test $\mathrm{P}<0.05$ ) (Supplementary Figure 1B) CD52 FC distributions varied significantly between the groups (Kruskal Wallis $\mathrm{P}<0.5$ ). Multiple comparisons, yet again, revealed no difference in Comparison 1 but a significant difference in Comparison 2 (Dunn's Post Test $\mathrm{P}<0.05$ ) (Supplementary Figure 1C). These results show firstly that no significant difference exists between the FC distributions of all the genes between the qPCRs (Comparison 1). Secondly, FC distributions of qPCRs and RNA-Seq do not show any difference for the upregulated genes ACSS3, SYDE1 and TBX10 (Comparison 2). However, for the downregulated genes, although no differences exist between qPCRs (Comparison 1); P2RY12 \& CD52 FC distributions differ significantly between qPCR and RNA-Seq. In summation, these results prove beyond reasonable doubt that differential expression assessed by qPCR using randomly chosen conventional reference genes and "stable" reference genes filtered from RNA-Seq render similar expression profiles of target genes without any significant difference among the two normalization strategies. However, concordance with RNA-Seq results was 


\section{Reference gene selection from RNA-Seq of mouse sciatic nerves (P3 vs P21)}

537 To rule out any circumstantial evidence that may support our observations in the iPSC dataset, 538 we sought to validate our results using a completely different experimental setting. We wanted 539 to rule out the possibility that the conventional reference genes that we chose in the iPSC 540 dataset were indeed stable reference genes by mere happenstance and thus they rendered the 541 same profiles of differential expression as the reference genes filtered from RNA-Seq.

542 Therefore, in a confirmatory experimental approach we mined and re-analyzed RNA-Seq data 543 that was previously generated from mouse sciatic nerves at Postnatal day 3 (P3) and P21 544 (Ackerman et al, 2018). This dataset permits us to study RNA expression changes in the sciatic 545 nerves during postnatal myelination. We applied the same criteria to select 10 candidate 546 reference genes (Ppp3ca, Fkbp4, Vcp, Lama2, Ank2, Coq9, Chmp2a, Laptm5, Leprotl1, 547 Supt $4 a$ ). The basemean values of these genes and other RNA-Seq data features are detailed in 548 Supplementary Table 2. nerves (P3 vs P21)

552 We evaluated the intrinsic intergroup variation of these reference genes by visual 553 representation of their non-normalized profiles $\left(2^{-\Delta \mathrm{Cq}}\right)$ using P3 samples as the calibrator 554 (Figure 4). As previously described, statistical inference was then assessed using the non555 parametric Mann Whitney U test owing to reduced sample sizes. Interestingly, and in contrast 556 to the iPSC dataset, 6 out of 10 genes (Fkbp4, Lama2, Coq9, Chmp2a, Leprotl1 \& Supt4a) 557 exhibit significant intergroup variation across the two experimental groups (Figure 4A, 4B, $558 \quad$ 4C, 4D, 4E \& 4F). 

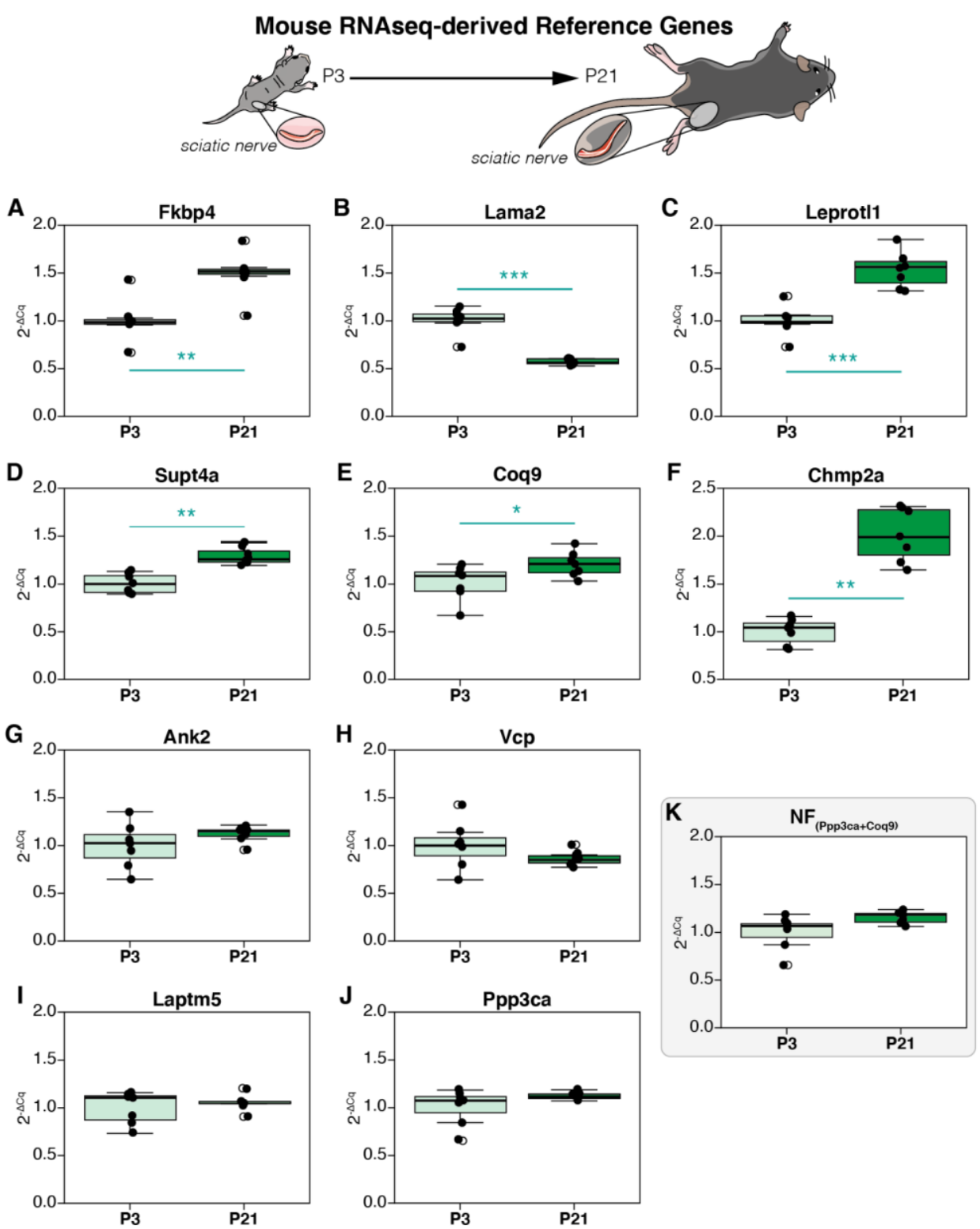

$560 \quad$ Figure 4. Raw expression profiles of reference genes obtained from RNA-Seq analysis of

561 developing sciatic nerve of age Post-natal day 3 (P5) and 21 (P21), expressed as fold changes (2- $\Delta \mathrm{Cq})$. P5 group is the experimental calibrator. (A) Fkbp4, (B) Lama2, (C) Leprotl4, (D) Supt4a, (E) Coq9, (F) Chmp2a, (G) Ank2, (H) Vcp, (I) Laptm5, (J) Ppp3ca, and (K)

564 Normalisation Factor (Ppp3ca + Coq9). Non-parametric Mann Whitney U test was used to assess differences between the groups. The alpha value was set at 0.05 and $P$ values are annotated as follows: $* P<0.05$, ** $P<0.01$, *** $P<0.001$.

567 We next performed the CV analysis to assess overall variation (Table 3). The genes tested 568 exhibited CV values between $12.32 \%$ and $36.24 \%$ and they were ranked as shown in Table 3.

569 As all genes exhibited $\mathrm{CV}<50 \%$, they could therefore be screened using the NormFinder 570 algorithm. NormFinder analysis further revealed Ppp3ca and Coq9 to be the best combination 571 of genes with a grouped Stability S score of 0.04 (Table 3). Although Coq9 exhibited 572 significant intergroup variation (Figure 4E), the combination of the gene with Ppp3ca 
resulting in the Normalization Factor (NF) does not exhibit any (Figure 4K). Interestingly, the RNA-Seq data features of the 6 genes that exhibit significant intergroup variation (Figure 4A, 4B, 4C, 4D, 4E \& 4F) show that they are not differentially expressed. However, if these genes were to be normalized using the NF computed herein, some of them could potentially exhibit differential expression in $\mathrm{qPCR}$ as the NF is stable across experimental groups (natural deduction, data not shown). It is a striking example to demonstrate the discordance of RNASeq and qPCR results in this dataset. However, the computation of a stable NF despite the presence of genes with significant intrinsic variation confirms the strength of the NormFinder algorithm and the validity of using the two best reference genes proposed by the method.

Table 3. CV and NormFinder analysis of reference genes derived from RNA-Seq

\begin{tabular}{|l|c|c|l|c|c|}
\hline \multicolumn{3}{|c|}{ CV Analysis } & \multicolumn{3}{c|}{ NormFinder } \\
\hline Gene & \%CV & Rank & Gene & Stability S & Rank \\
\hline Laptm5 & 12.32 & 1 & Ank2 & 0.07 & 1 \\
\hline Ppp3ca & 12.96 & 2 & Ppp3ca & 0.08 & 2 \\
\hline Supt4a & 14.79 & 3 & Coq9 & 0.08 & 3 \\
\hline Coq9 & 16.26 & 4 & Laptm5 & 0.16 & 4 \\
\hline Ank2 & 16.31 & 5 & Supt4a & 0.16 & 5 \\
\hline Vcp & 19.59 & 6 & Leprotl1 & 0.23 & 6 \\
\hline Leprotl1 & 24.49 & 7 & Fkbp4 & 0.26 & 7 \\
\hline Fkbp4 & 25.86 & 8 & Vcp & 0.27 & 8 \\
\hline Lama2 & 29.96 & 9 & Chmp2a & 0.47 & \\
\hline Chmp2a & 36.24 & Lama2 & & \\
\hline
\end{tabular}




\section{Reference gene validation of conventional mouse reference genes (P3 vs P21)}

589 To obtain stable reference genes from a set of conventional genes, we used 10 candidate mouse 590 reference genes that we had previously used to establish our workflow (Actb, Gapdh, Tbp, 591 Sdha, Pgk1, Ppia, Rpl13a, Hsp60, Mrpl10, Rps26) (Sundaram et al, 2019). As explained above, 592 visual representation followed by inferential statistics were performed and the results are 593 described in Figure 5. 4 out of 10 genes (Gapdh, Sdha, Pgk1 \& Rps26) exhibited significant 594 intrinsic variation between the experimental groups (Figure 5G ,5H, 5I \& 5J)
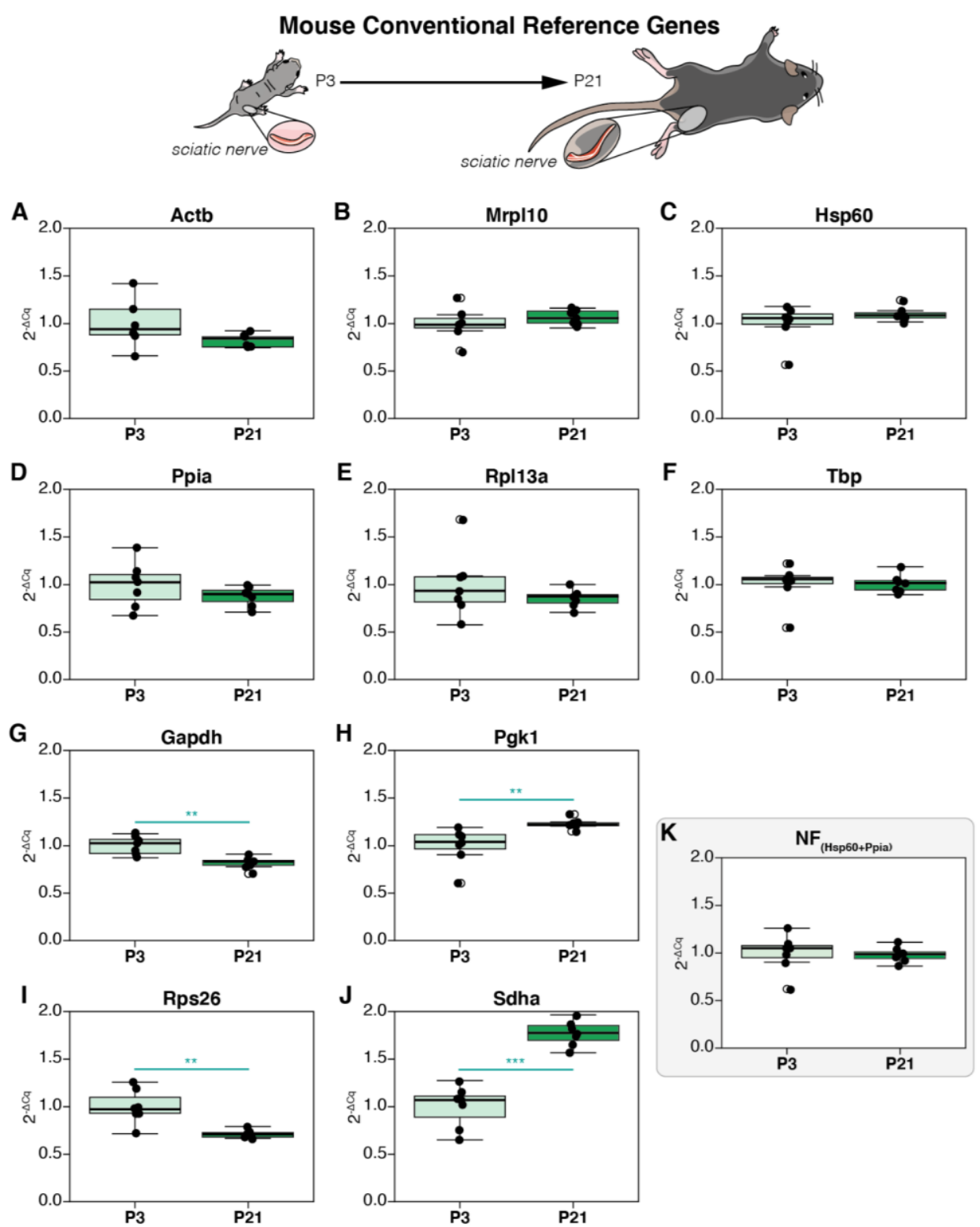

596 Figure 5. Raw expression profiles of conventional reference genes of developing sciatic 597 nerve of age Post-natal day 3 (P3) and 21 (P21), expressed as fold changes (2-4Cq). P3 group 598 is the experimental calibrator. (A) Actb, (B) Mrpl10, (C) Hsp60, (D) Ppia, (E) Rpl13a, (F) 599 Tbp, (G) Gapdh, (H) Pgk1, (I) Rps26, (J) Sdha, and (K) Normalisation Factor (Hsp60 + Ppia).

600 Non-parametric Mann Whitney U test was used to assess differences between the groups. The 
601 alpha value was set at 0.05 and $P$ values are annotated as follows: $* P<0.05$, ** $P<0.01$, ***

$602 \quad P<0.001$

$603 \mathrm{CV}$ analysis was then performed on these genes and the results are depicted in Table 4. CV 604 values ranged from $12.3 \%$ to $30.44 \%$ and the genes were ranked accordingly. As all genes 605 exhibited $\mathrm{CV}<50 \%$, we screened these genes through the NormFinder algorithm to determine 606 the best combination of reference genes for qPCR normalization. NormFinder determined 607 Hsp60 and Ppia to be the best combination of reference genes with a grouped Stability S Score 608 of 0.06 (Table 6). Both Hsp60 (Figure 5C) \& Ppia (Figure 5D) did not exhibit any intergroup 609 variation and the same was true for the NF computed by combining these genes (Figure 5K).

Table 4. CV and NormFinder analysis of conventional reference genes

611

\begin{tabular}{|c|c|c|c|c|c|}
\hline \multicolumn{3}{|c|}{ CV Analysis } & \multicolumn{3}{|c|}{ NormFinder } \\
\hline Gene & $\% \mathrm{CV}$ & Rank & Gene & Stability $S$ & Rank \\
\hline Mrpl10 & 12.30 & 1 & $T b p$ & 0.03 & 1 \\
\hline Gapdh & 13.06 & 2 & Mrpl10 & 0.04 & 2 \\
\hline Hsp60 & 14.36 & 3 & Hsp60 & 0.05 & 3 \\
\hline$T b p$ & 15.24 & 4 & Ppia & 0.05 & 4 \\
\hline$P g k 1$ & 15.83 & 5 & Pgkl & 0.06 & 5 \\
\hline Ppia & 19.36 & 6 & $A c t b$ & 0.07 & 6 \\
\hline$A c t b$ & 21.07 & 7 & Rpl13a & 0.10 & 7 \\
\hline Rps26 & 22.04 & 8 & Gapdh & 0.11 & 8 \\
\hline Rpl13a & 26.78 & 9 & Rps26 & 0.14 & 9 \\
\hline Sdha & 30.44 & 10 & Sdha & 0.18 & 10 \\
\hline
\end{tabular}

612

613 Normfinder Best combination: Hsp60 + Ppia

614 Grouped stability: 0.06

615 


\section{Differential expression of target genes by qPCR and RNA-Seq (P3 vs P21)}

617 Following reference gene validation of conventional and RNAseq-derived candidates, we 618 picked 6 target genes that were known to be differentially expressed during postnatal 619 development of the sciatic nerve (Gerber et al, 2021). 3 target genes (Mpz, Mbp and Cd90) that 620 were chosen are significantly upregulated and the other 3 target genes ( $p 75 N T R, M k i 67$ and 621 Sox2) are significantly downregulated at these time points. The relative expression of these 622 genes was then computed by qPCR using the Normalization Factor (NF) from conventional 623 candidate reference genes (Hsp60 \& Ppia or Conventional NF) or from RNAseq-derived 624 candidate reference genes ( $P p p 3 c a$ \& Coq9 or RNAseq derived NF) (Figure 6). To compare 625 our qPCR results, we also computed the fold change assessed by RNA-Seq using the 626 Normalized counts of these genes.

627 Regarding the downregulated genes, Mki67 expression in the P21 group, when assessed 628 through qPCRs, exhibited fold change values of $0.14 \pm 0.10$ (mean FC \pm SD, conventional NF, 629 Figure 6A) and $0.12 \pm 0.08$ (RNA-Seq derived NF, Figure 6B). In RNA-Seq, the fold change 630 values were $0.08 \pm 0.01$ (Figure 6C). p75NTR expression fold change in the P21 group were $6310.30 \pm 0.04$ (conventional NF, Figure 6D) and $0.26 \pm 0.02$ (RNA-Seq derived NF, Figure 6E) 632 when assessed using qPCRs. However, RNA-Seq fold change values of $p 75 N T R$ were 633 comparatively higher at $0.63 \pm 0.18$ (Figure 6F). Sox 2 expression fold change in the P21 group 634 when assessed by qPCR were $0.49 \pm 0.05$ (Conventional NF, Figure 6G) and $0.42 \pm 0.03$ 635 (RNA-Seq derived NF, Figure 6H). RNA-Seq changes were at $0.39 \pm 0.09$ (Figure 6I).

636 For the upregulated genes, $M b p$ expression levels in the P21 group exhibited fold change values 637 of $1.73 \pm 0.15$ (Conventional NF, Figure 6J) and $1.49 \pm 0.19 \mathrm{~s}$ (RNA-Seq derived NF, Figure 638 6K). The fold changes in RNA-Seq were $2.02 \pm 0.62$ (Figure 6L). $M p z$ expression levels 639 assessed by qPCR were at $1.76 \pm 0.38$ (Conventional NF, Figure 6M) and $1.53 \pm 0.35$ (RNA640 Seq derived NF, Figure 6N). However, RNA-Seq expression fold change of $M p z$ were 641 significantly higher at $3.49 \pm 0.26$ (Figure 60). Finally, $C d 90$ expression fold change assessed 642 by qPCR were at $1.83 \pm 0.35$ (Conventional NF, Figure 6P) and $1.57 \pm 0.30$ (RNA-Seq derived 643 NF, Figure 6Q). RNA-Seq fold changes were at $4.81 \pm 2.77$ (Figure 6R). Yet again, these 644 results taken together with the visual representation of differential expression described in 645 Figure 6 show that qPCR normalization using conventional NF or RNA-Seq derived NF render 646 very similar results. We observe again that they do not always concur with RNA-Seq fold 647 changes in magnitude, but they do so in tendency. 

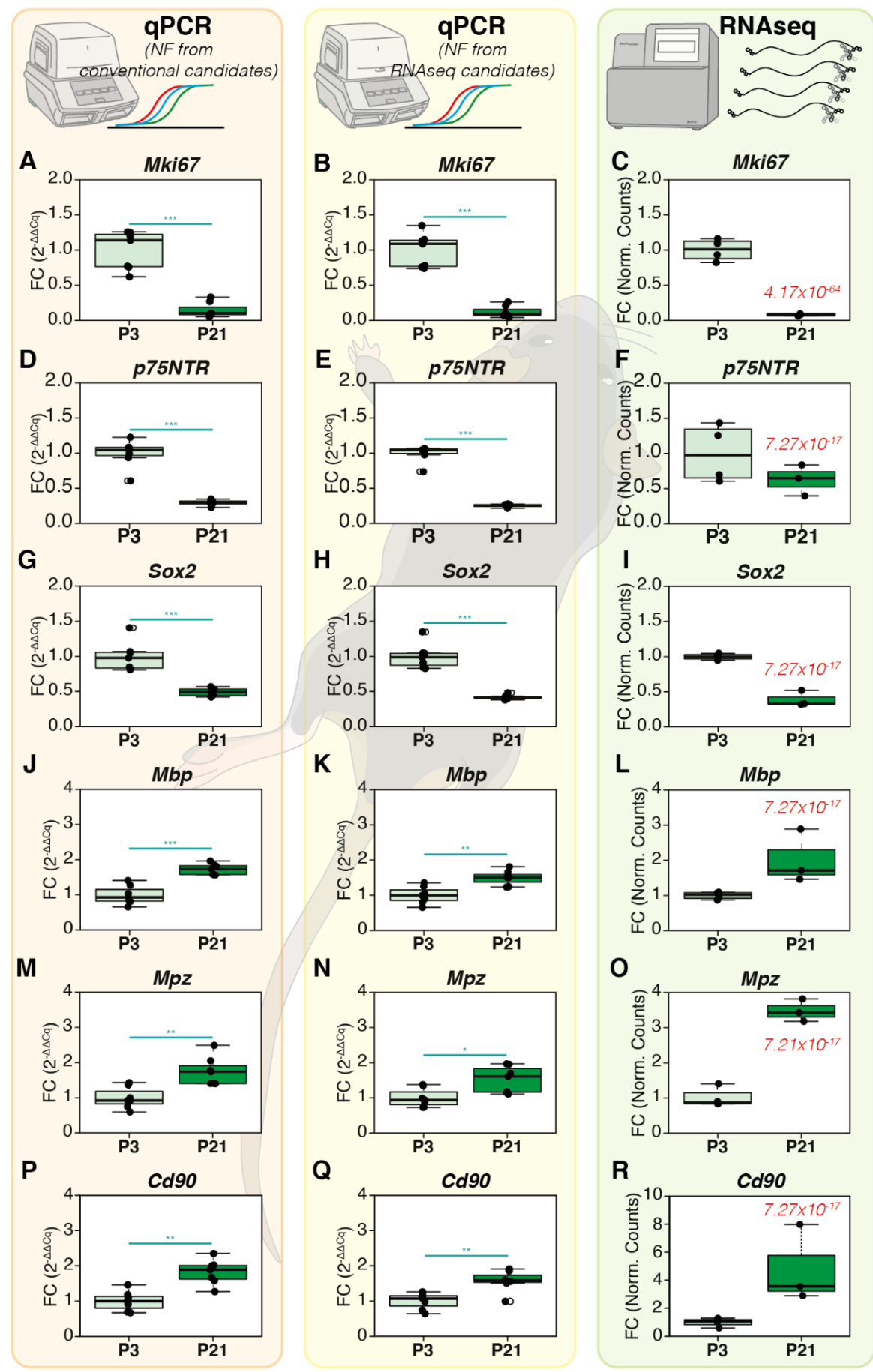

649 Figure 6: Differential expression of target genes in P3 and P21 mouse sciatic nerves by

650 qPCRs and RNA-Seq. The target genes (Mki67, p75NTR, Sox2, Mbp, Mpz, Cd90) have been 651 normalized with the normalization factor $(N F)$ computed from conventional reference genes 652 Hsp60 \& Ppia in the first column $(A, D, G, J, M, P)$ or from RNA-Seq derived reference genes 653 Ppp3ca and Coq9 in the second column $(B, E, H, K, N, Q)$. The third column contains the Fold 654 Changes computed from RNA-Seq $(C, F, I, L, O, R)$. The normalized counts for each gene in each 655 group were used to calculate the fold changes in RNA-Seq. The WT group was used as the 656 experimental control. The $P_{\text {adj }}$ value of RNA-Seq is indicated in the P21 group for each gene 
657 in the RNA-Seq column. For the qPCRs, Non-parametric Mann Whitney U test was used to

658 assess differences between the groups. The alpha value was set at 0.05 and $P$ values are 659 annotated as follows: $* P<0.05$, ** $P<0.01$, *** $P<0.001$.

\section{Comparison of differential expression between qPCRs and RNA-Seq (P3 vs P21):}

661 Similar to the analysis performed in the iPSC dataset, we next investigated in detail if the Fold 662 Change distributions in the P21 experimental group computed across the 3 methods differed 663 significantly from one another. We used non-parametric ANOVA (Kruskal Wallis Test) of 664 ordinal distributions followed by Dunn's multiple comparison post-test (Supplementary 665 Figure 2). As shown in the iPSC dataset, we performed multiple comparisons between qPCRs 666 (Comparison 1) and also between qPCR and RNA-Seq (Comparison 2).

667 Non-parametric ANOVA revealed no significant difference between the FC distributions of $668 M k i 67$ and $M b p$ (Supplementary Figure 2A and 2E) suggesting that the qPCR fold changes 669 concur with RNA-Seq fold changes in these genes. Sox2, p75NTR and Mpz FC distributions 670 varied significantly between the groups (Kruskal Wallis $\mathrm{P}<0.05, \mathrm{P}<0.01$ and $\mathrm{P}<0.01$ 671 respectively); however, Dunn's post test revealed no significant difference in both Comparison 6721 and Comparison 2 (Supplementary Figure 2B, 2C \& 2F). Cd90 FC distributions varied 673 significantly between the groups (Kruskal Wallis $\mathrm{P}<0.01$ ). Multiple comparisons revealed no 674 difference in Comparison 1 and Comparison 2 (Supplementary Figure 2D). In summation, 675 all the genes tested in the sciatic nerve dataset show no significant differences between 676 Conventional NF and RNA-Seq-derived NF results. In summation, these results show once 677 again that stable reference genes filtered from RNA-Seq data do not give any added advantage 678 to qPCR data normalization and that not all genes exhibit comparable Fold Changes when 679 comparing qPCR results and RNA-Seq. 


\section{Discussion:}

682

683

684

685

686

687

688

689

690

691

692

693

694

695

696

697

698

699

700

701

702

703

704

705

706

707

708

709

710

711

712

713

714

715

716

717

718

719

720

721

722

The central premise of this article was to demonstrate that stable reference genes for qPCR data normalisation can be obtained from any random set of candidates provided the statistical approach of reference gene validation is sound and consistent. To this end, using our qPCR workflow we have shown using two separate and unique datasets that randomly chosen reference genes can render the same results of differential expression when compared to stable references selected from RNA-Seq data.

The advantage of this approach in filtering the best candidates from any random set lies in combining 3 methods that are complementary to each other. Although multiple statistical methods have been proposed before, we have previously highlighted the potential pitfalls and assumptions of these computational methods (Sundaram et al, 2019). We thus devised this workflow combining the well-known NormFinder method with CV analysis and the visual representation of non-normalized expression followed by statistical testing. Indeed, it should be noted the core method of this approach is the NormFinder algorithm. However, this method was first described and validated with larger sample sizes (typically between 10 - 20 or more) and with a high number of candidate genes chosen from microarrays (typically between 15 20) exhibiting very little overall variation (Andersen et al, 2004). In our previous study, we identified that lower sample sizes and candidate genes with high overall variation can skew the results of this method because of the algorithm's construct (Sundaram et al, 2019). Although scores of studies have used this algorithm without due diligence to the prerequisites, our combined approach resolves these issues. The CV analysis helps in the identification of genes with high overall variance and their successive elimination from analysis gives rise to more robust results from NormFinder. Visual representation of the non-normalized profiles $\left(2^{-\Delta \mathrm{Cq}}\right)$ followed by statistical testing further validates the results of NormFinder as the Normalization Factor computed from the two best reference genes does not exhibit any significant differences between the experimental groups regardless of experimental conditions (Figure 1K, 2K, 4K $\boldsymbol{\&} \mathbf{5 K})$. Thus, in combining CV analysis and statistical testing of non-normalized profiles, we extrapolated the NormFinder approach to be used for experimental setups involving lower sample sizes similar to those used in this study.

The cut off at $50 \% \mathrm{CV}$ to qualify for NormFinder analysis is rooted in the fact that at $50 \%$, the standard deviation of the expression distribution is half the mean expression level. Previous datasets that we published have indicated that the Normalization Factor (NF) computed by NormFinder when including genes that exhibit CV>50\% exhibit significant intergroup variation in the non-normalized profiles (Sundaram et al, 2019). Below this cut off, $\mathrm{NF}$ expression levels are stable across experimental groups. Indeed, the user can be more stringent by using a lower CV cut off but our data from this study and the previous one suggests that this is not necessary. However, this criterion gives rise to a theoretical limitation of our approach. In a given experimental setup, if all the candidate reference genes tested exhibit CV values of above $50 \%$, then our approach will fail and NormFinder results would no longer be reliable. In such cases, a new list of candidates is required. However, our experience suggests 
that as little as 6 genes after eliminating genes exhibiting $\mathrm{CV}>50 \%$ can still provide a sound NF for qPCR data normalization (Sundaram et al, 2019).

Although not the primary objective of the study, the comparison of qPCR results with RNA-Seq merits discussion. In the iPSC dataset, comparisons made between qPCR and RNASeq results revealed that 4 out of 6 genes (ACSS3, SYDE1, TREM2 and TBX10) showed no significant difference between the fold changes computed by both the methods (Supplementary Figures 1D, 1E, 1F \& 1C). However, changes in the expression of CD52 and $P 2 R Y 12$ significantly differed between the two methods (Figure 3 and Supplementary Figure 1A and 1B). In the sciatic nerve dataset, 5 out of 6 genes tested show no difference between qPCR and RNA-Seq data in multiple comparisons (Supplementary Figure 2A, 2B, 2C, 2E, 2F). However, this analysis is not optimal as the number of samples used in qPCR is much higher $(\mathrm{N}=7)$ than the number of samples in RNA-Seq data that we mined $(\mathrm{N}=3)$. In reality, only 3 genes (Mki67, Sox2 \& Mbp) exhibited overlapping fold change distributions across the methods (Supplementary Figure 2A, 2B \& 2E). However, regardless of the magnitudes of the change observed, the pattern of expression changes was always conserved across qPCR and RNA-Seq for the genes that we tested. Indeed, it is possible that if we include more target genes, we can potentially notice disagreements in expression patterns as well. However, as stated earlier, our objective is restricted to demonstrating the futility of selecting reference genes from RNA-Seq data for qPCR data normalization. The comparison of the two methodologies falls beyond the scope of this study but it has been amply addressed elsewhere (Griffith et al, 2010; Rajkumar et al, 2015; Everaert et al, 2017).

These studies have indeed tried to validate RNA-Seq data with RTqPCR for a large set of target genes typically ranging from a few hundred to the entire transcriptome. The consensus from these studies is that the extent of correlation between RTqPCR and RNAseq data is dependent on how the sequencing data was aligned, mapped and counted based on existing protocols. Additionally, though not discussed in these articles, we believe that this would equally depend on how reference genes were selected for qPCR assays that validate the RNAseq data. Overlooking these technicalities, these studies also show that around $85 \%$ of all genes tested show concordant differential expression (comparable magnitudes with the same tendency) between the two methodologies with correlation coefficients typically above 0.8 . Although these numbers are relatively reassuring at the outset, from an absolute perspective, the implications raise important concerns. For a hypothetical bulk RNA-Seq dataset of around 18000 protein-coding transcripts, $85 \%$ concordance leaves 2700 genes whose differential expression is discordant between RNA-Seq and qPCR. Of note, the study conducted by Everaert and colleagues (Everaert et al, 2017) conclude that discordant genes typically are smaller, have fewer exons and are expressed at lower levels. Indeed, and as mentioned in the introduction, these observations can be possibly substantiated by the transcript-length bias and the bias against genes with lower expression in RNA-Seq. In our study, the discordant genes CD52 and P2RY12 in the microglia dataset have only 2 exons. The other 4 concordant genes have between $4-13$ exons and therefore are relatively larger. The basemean values (and therefore the expression levels) of the 2 discordant genes are largely higher than the 4 concordant genes. Therefore, number of exons appears to be more important than expression 
767 levels for concordance between qPCR and RNA-Seq for these target genes. On the contrary,

768 we did not observe any correlation between discordance and small gene size or lower 769 expression in the sciatic nerve samples. These observations show that an objective approach to

770 identify discordant genes is lacking in literature. As RNA-Seq data analysis is particularly 771 prone to certain biases while computing differential gene expression, it would be valuable to 772 computationally suggest qPCR validation for specific genes that are susceptible to these 773 normalization biases based on their transcript length, expression levels and other unidentified 774 parameters. Ideally, these suggestions should be integrated into the data analysis pipelines and 775 represented in the differential gene expression data frames. This would provide the foundation 776 for an objective and structured validation of Sequencing data rather than abtuse overall 777 validation of RNA-Seq results by qPCR which is indeed burdensome. If these computational 778 suggestions are achieved, the methodology used in our study would aptly address the necessity 779 of performing robust and reproducible qPCR assays using any random set of reference genes 780 that are independent of RNA-Seq results. 


\section{References:}

Ackerman SD, Luo R, Poitelon Y, Mogha A, Harty BL, D'Rozario M, Sanchez NE, Lakkaraju AKK, Gamble P, Li J, et al (2018) GPR56/ADGRG1 regulates development and maintenance of peripheral myelin. $J$ Exp Med 215: 941-961

Andersen C, Jensen J \& Orntoft T (2004) Normalization of Real - Time Quantitative Reverse Transcription - PCR Data : A Model - Based Variance Estimation Approach to Identify Genes Suited for Normalization, Applied to Bladder and Colon Cancer Data Sets. Cancer Res 64: 5245

Andreone BJ, Przybyla L, Llapashtica C, Rana A, Davis SS, van Lengerich B, Lin K, Shi J, Mei Y, Astarita G, et al (2020) Alzheimer's-associated PLC $\gamma 2$ is a signaling node required for both TREM2 function and the inflammatory response in human microglia. Nat Neurosci 23: 927-938

Berge K Van den, Hembach KM, Soneson C, Tiberi S, Clement L, Love MI, Patro R \& Robinson MD (2019) RNA Sequencing Data: Hitchhiker's Guide to Expression Analysis. https://doi.org/101146/annurev-biodatasci-072018-021255 2: 139-173

Boda E, Pini A, Hoxha E, Parolisi R \& Tempia F (2009) Selection of Reference Genes for Quantitative Real-time RT-PCR Studies in Mouse Brain. J Mol Neurosci 37: 238-253

Bustin SA, Benes V, Garson JA, Hellemans J, Huggett J, Kubista M, Mueller R, Nolan T, Pfaffl MW, Shipley GL, et al (2009) The MIQE Guidelines: Minimum Information for Publication of Quantitative Real-Time PCR Experiments.

Carmona R, Arroyo M, Jiménez-Quesada MJ, Seoane P, Zafra A, Larrosa R, Alché J de D \& Claros MG (2017) Automated identification of reference genes based on RNA-seq data. Biomed Eng Online 16

Chapman JR \& Waldenström J (2015) With Reference to Reference Genes: A Systematic Review of Endogenous Controls in Gene Expression Studies. PLoS One 10: e0141853

Chim SSC, Wong KKW, Chung CYL, Lam SKW, Kwok JSL, Lai C-Y, Cheng YKY, Hui ASY, Meng M, Chan O-K, et al (2017) Systematic Selection of Reference Genes for the Normalization of Circulating RNA Transcripts in Pregnant Women Based on RNA-Seq Data. Int J Mol Sci 18: 1709

Coenye T (2021) Do results obtained with RNA-sequencing require independent verification? Biofilm 3: 100043

Dai Y, Cao Y, Köhler J, Lu A, Xu S \& Wang H (2021) Unbiased RNA-Seq-driven identification and validation of reference genes for quantitative RT-PCR analyses of pooled cancer exosomes. BMC Genomics 22: 27

Durinck S, Spellman PT, Birney E \& Huber W (2009) Mapping Identifiers for the Integration of Genomic Datasets with the R/Bioconductor package biomaRt. Nat Protoc 4: 1184

Eisenberg E \& Levanon EY (2013) Human housekeeping genes, revisited. Trends Genet 29: 569-74

Everaert C, Luypaert M, Maag JL V, Cheng QX, Dinger ME, Hellemans J \& Mestdagh P (2017) Benchmarking of RNA-sequencing analysis workflows using wholetranscriptome RT-qPCR expression data. Sci Rep 7: 1559

Gerber D, Pereira JA, Gerber J, Tan G, Dimitrieva S, Yángüez E \& Suter U (2021) Transcriptional profiling of mouse peripheral nerves to the single-cell level to build a sciatic nerve atlas (Snat). Elife 10

Griffith M, Griffith OL, Mwenifumbo J, Goya R, Morrissy AS, Morin RD, Corbett R, Tang MJ, Hou YC, Pugh TJ, et al (2010) Alternative expression analysis by RNA sequencing. Nat Methods 7: 843-847

Haenseler W, Sansom SN, Buchrieser J, Newey SE, Moore CS, Nicholls FJ, Chintawar S, Schnell C, Antel JP, Allen ND, et al (2017) A Highly Efficient Human Pluripotent Stem 
Cell Microglia Model Displays a Neuronal-Co-culture-Specific Expression Profile and Inflammatory Response. Stem Cell Reports 8: 1727-1742

Hernandez-Segura A, Rubingh R \& Demaria M (2019) Identification of stable senescenceassociated reference genes. Aging Cell 18: e12911

Hoang VLT, Tom LN, Quek X-C, Tan J-M, Payne EJ, Lin LL, Sinnya S, Raphael AP, Lambie D, Frazer IH, et al (2017) RNA-seq reveals more consistent reference genes for gene expression studies in human non-melanoma skin cancers. PeerJ 5: e3631

Jacob F, Guertler R, Naim S, Nixdorf S, Fedier A, Hacker NF \& Heinzelmann-Schwarz V (2013) Careful Selection of Reference Genes Is Required for Reliable Performance of RT-qPCR in Human Normal and Cancer Cell Lines. PLoS One 8: e59180

Liu Y, Zhou J \& White KP (2014) RNA-seq differential expression studies: more sequence or more replication? Bioinformatics 30: 301-304

Livak KJ \& Schmittgen TD (2001) Analysis of relative gene expression data using real-time quantitative PCR and the $2^{\wedge}-\Delta \Delta C \mathrm{~T}$ Method. Methods 25: 402-408

Love MI, Huber W \& Anders S (2014) Moderated estimation of fold change and dispersion for RNA-seq data with DESeq2. Genome Biol 20141512 15: 1-21

Mandelboum S, Manber Z, Elroy-Stein O \& Elkon R (2019) Recurrent functional misinterpretation of RNA-seq data caused by sample-specific gene length bias. PLOS Biol 17: e3000481

McQuade A, Kang YJ, Hasselmann J, Jairaman A, Sotelo A, Coburn M, Shabestari SK, Chadarevian JP, Fote G, Tu CH, et al (2020) Gene expression and functional deficits underlie TREM2-knockout microglia responses in human models of Alzheimer's disease. Nat Commun 11: 1-17

Mollet IG, Ben-Dov C, Felício-Silva D, Grosso AR, Eleutério P, Alves R, Staller R, Silva TS \& Carmo-Fonseca M (2010) Unconstrained mining of transcript data reveals increased alternative splicing complexity in the human transcriptome. Nucleic Acids Res 38: 4740-4754

Oshlack A \& Wakefield MJ (2009) Transcript length bias in RNA-seq data confounds systems biology. Biol Direct 200941 4: 1-10

Pfaffl MW, Tichopad A, Prgomet C \& Neuvians TP (2004) Determination of stable housekeeping genes, differentially regulated target genes and sample integrity: BestKeeper-Excel-based tool using pair-wise correlations. Biotechnol Lett 26: 509-515

Pombo MA, Zheng Y, Fei Z, Martin GB \& Rosli HG (2017) Use of RNA-seq data to identify and validate RT-qPCR reference genes for studying the tomato- Pseudomonas pathosystem. Sci Rep 7: 44905

Rajkumar AP, Qvist P, Lazarus R, Lescai F, Ju J, Nyegaard M, Mors O, Børglum AD, Li Q \& Christensen JH (2015) Experimental validation of methods for differential gene expression analysis and sample pooling in RNA-seq. BMC Genomics 16: 1-8

Schmittgen TD \& Livak KJ (2008) Analyzing real-time PCR data by the comparative C(T) method. Nat Protoc 3: 1101-8

Silver N, Best S, Jiang J \& Thein SL (2006) BMC Molecular Biology Selection of housekeeping genes for gene expression studies in human reticulocytes using real-time PCR.

De Spiegelaere W, Dern-Wieloch J, Weigel R, Schumacher V, Schorle H, Nettersheim D, Bergmann M, Brehm R, Kliesch S, Vandekerckhove L, et al (2015) Reference gene validation for RT-qPCR, a note on different available software packages. PLoS One 10: $1-13$

Sundaram VK, Sampathkumar NK, Massaad C \& Grenier J (2019) Optimal use of statistical methods to validate reference gene stability in longitudinal studies. PLoS One 14: e0219440 
bioRxiv preprint doi: https://doi.org/10.1101/2021.08.21.457202; this version posted August 25, 2021. The copyright holder for this preprint (which was not certified by peer review) is the author/funder, who has granted bioRxiv a license to display the preprint in perpetuity. It is made available under aCC-BY-NC-ND 4.0 International license.

881 Thellin O, Zorzi W, Lakaye B, De Borman B, Coumans B, Hennen G, Grisar T, Igout A \& Heinen E (1999) Housekeeping genes as internal standards: use and limits (2002) Accurate normalization of real-time quantitative RT-PCR data by geometric averaging of multiple internal control genes. Genome Biol 3: RESEARCH0034

Yang H, Liu J, Huang S, Guo T, Deng L \& Hua W (2014) Selection and evaluation of novel reference genes for quantitative reverse transcription PCR (qRT-PCR) based on genome and transcriptome data in Brassica napus L. Gene 538: 113-122

Zhan C, Zhang Y, Ma J, Wang L, Jiang W, Shi Y \& Wang Q (2014) Identification of reference genes for qRT-PCR in human lung squamous-cell carcinoma by RNA-Seq. Acta Biochim Biophys Sin (Shanghai) 46: 330-337

Zhou Z, Cong P, Tian Y \& Zhu Y Using RNA-seq data to select reference genes for normalizing gene expression in apple roots. 